\title{
Traumatic Flap Dislocation and Management After 17 Years of Lasik Surgery
}

\section{Arun Muppliyath Raghavan*}

Optometrist, Department of Ophthalmology, Mediclinic Airport Road Hospital, Abu

Dhabi, UAE

*Corresponding Author: Arun Muppliyath Raghavan, Optometrist, Department of Ophthalmology, Mediclinic Airport Road Hospital, Abu Dhabi, UAE.
Received: November 09, 2021

Published: November 15, 2021

(C) All rights are reserved by Arun Muppliyath Raghavan.

\section{Abstract}

LASIK flap dislocation due to trauma is an uncommon uncomplication that can occur at any time after surgery, with a potential of being visually debilitating. In this case report, we describe the occurrence and subsequent management of a traumatic flap dislocation 17 years after surgery.

Keywords: LASIK; PRK; Corneal Flap Complications; Late Corneal Flap Dislocation; Femtosecond-assisted LASIK

\section{Abbreviations}

LASIK: Laser Assisted In Situ Keratomileusis; PRK: Photorefractive Keratectomy; RE: Right Eye; LE: Left Eye; IOP: Intra Ocular Pressure; OCT: Optical Coherence Tomography

\section{Introduction}

LASIK (Laser Assisted in-situ Keratomileusis) is a refractive surgery for the correction of refractive errors such as myopia, hyperopia, and astigmatism involving the creation of a corneal flap and reshaping of underlying stroma with an excimer laser to correct the refractive error. Patients who undergo LASIK achieve faster visual rehabilitation and less post-operative discomfort when compared to surface ablation techniques such as PRK $[1,2]$. The flap created in LASIK is susceptible to dislocation after blunt trauma even after a number of years postoperatively. We describe a case of post traumatic flap dislocation and its management 17 years after LASIK Surgery.

\section{Case History}

A 34-year-old female who underwent microkeratome LASIK in both eyes was referred to our practice, having sustained injury to her right eye with a soda bottle cap 8 days back. She was initially misdiagnosed as a corneal abrasion and was treated with a ban- dage contact lens for 4 days, to no effect. She was then referred to a corneal specialist after failure of treatment.

On clinical examination, the Visual Acuity was found to be 20/200 and 20/20 for RE and LE respectively. The auto refraction readings and IOP were not measured as patient was having severe pain. In the slit lamp examination for the right eye, the cornea showed am inferior traumatic flap dislocation (Figure 1a) with a strand of debris in the interface. Diffuse lamellar keratitis and mild epithelial ingrowth were also noted. Examination of the left eye was normal.

Optical coherence tomography of the right cornea showed the LASIK Flap folded over in the interface (Figure 1b).

Collection of corneal cultures, LASIK flap repositioning, antibiotic irrigation, epithelial debridement of the stromal bed and beyond the flap margins was performed immediately. A bandage contact lens was inserted at the end of procedure and kept in place for 2 weeks. The procedure was uneventful, and patient was advised for a follow up after 2 days. The corneal culture results were negative. The patient was placed on hourly Moxifloxacin and Prednisolone acetate eye drops. 


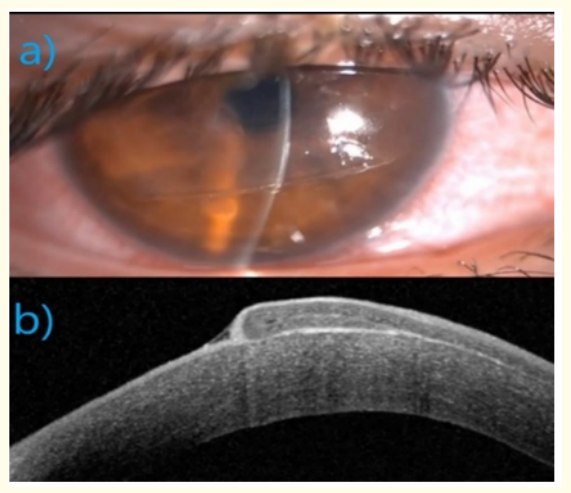

Figure 1: a) Anterior segment photograph showing dislocated LASIK flap in the inferior part of cornea.

b) Anterior segment OCT showing inferiorly dislocated LASIK flap folded over the stromal bed in the interface.

Diffuse lamellar keratitis (DLK) was noted at the first postoperative visit after 2 days, which had resolved by 1 week. The Visual acuity of the right eye at 1 week had improved to 20/25 and the auto refractometer readings were -1.00DS /-1.00DC x30. On slit lamp examination the LASIK flap had settled back into place (Figure $2 \mathrm{a}$ and $\mathrm{b}$ ) with mild central haze remaining as a sequel of the diffuse lamellar keratitis. Mild irregular astigmatism was seen on corneal topography (Figure 3).
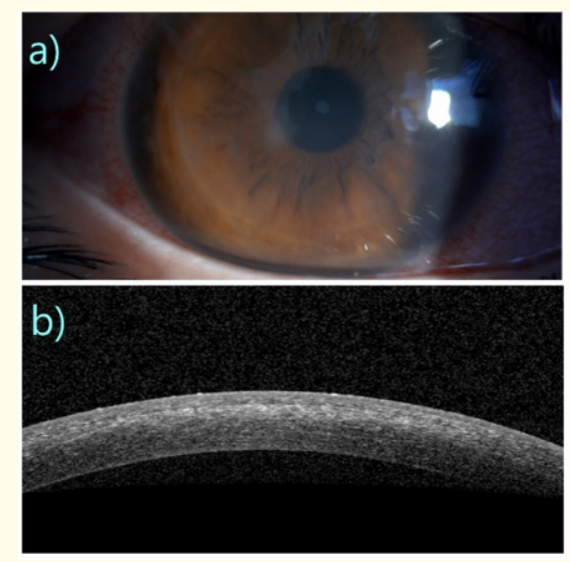

Figure 2: a) Anterior segment photo showing LASIK flap repositioned in place.

b) Anterior segment OCT showing LASIK flap in position and secondary corneal haze.

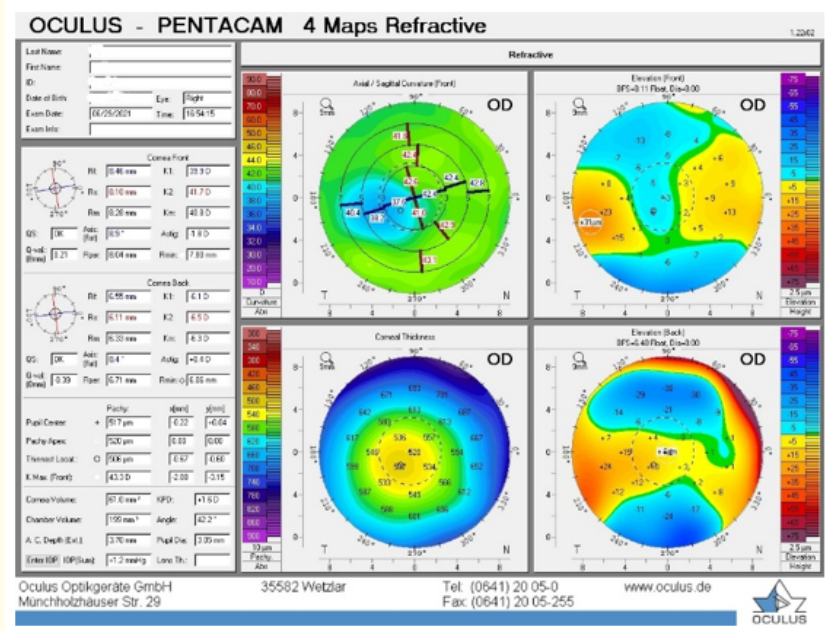

Figure 3: Corneal topography showing secondary irregular Astigmatism.

At 1 month, the patients' unaided vision had further improved to $20 / 20$ - with auto refractor readings of - $0.75 \mathrm{DC} \times 5$, with further regularisation of the corneal topography. Some focal flattening was noted corresponding to the area of post DLK haze in the cornea. At this point the patient was advised to stop their medications and discharged from clinic with subsequent follow-up as needed.

\section{Discussion}

Traumatic LASIK flap dislocation is an uncommon, but well documented complication after LASIK surgery. To date the longest reported duration between surgery and dislocation ranges between 13 or 14 years $[3,4]$. This case, with the dislocation occurring 17 years after surgery, is significant in being the longest reported interval in literature. It is also significant in that spite of a delay in diagnosis and appropriate treatment a good visual outcome was achieved. This case demonstrates that the biomechanical strength of post LASIK corneas will never attain that of normal untreated corneas $[5,6]$.

\section{Conclusion}

Post LASIK corneas are at a potential risk of traumatic corneal flap dislocation even many years after surgery. Appropriate case selection for LASIK, excluding individuals prone to blunt trauma (e.g., contact sports, uniformed services), and proper postopera- 
tive counselling, explaining the potential for flap dislocation to LASIK patients can help decrease the incidence of traumatic flap dislocation. Fortunately, with Proper Surgical intervention and management, the visual prognosis is good in such cases.

\section{Acknowledgement}

The completion of this case report could not have been possible with the help of Dr. Hamed Anwar, Consultant Ophthalmologist, Mediclinic Airport Road Hospital, Abu Dhabi, UAE. His contributions are sincerely appreciated and gratefully acknowledged.

I would also like to thank my colleagues in Optometry Department and the whole Ophthalmology team in Mediclinic Airport Road Hospital, Abu Dhabi, UAE for their endless support.

\section{Bibliography}

1. Hatch BB., et al. "A prospective, contralateral comparison of photorefractive keratectomy (PRK) versus thin-flap LASIK: assessment of visual function". Clinical Ophthalmology 5 (2011): 451-457.

2. Hashemi H., et al. "Femtosecond-assisted LASIK versus PRK: comparison of 6-month visual acuity and quality outcome for high myopia". Eye Contact Lens 42.6 (2016): 354-357.

3. DG Holt. Surgical management of traumatic LASIK flap dislocation with macrostriae and epithelial ingrowth 14 years postoperatively". Journal of Cataract and Refractive Surgery 38 (2012): 357-361.

4. Galvis V., et al. "Traumatic corneal flap avulsion and loss 13 years after LASIK". Saudi Journal of Ophthalmology 33.2 (2019): 172-176.

5. Schmack DG., et al. "Cohesive tensile strength of human LASIK wounds with histologic ultrastructural, and clinical correlations". Journal of Refractive Surgery 21 (2005): 433-445.

6. V Galvis., et al. "Risk factors and visual results in cases of LASIK flap repositioning due to folds or dislocation: case series and literature review". International Ophthalmology 34 (2014): 1926.

Volume 4 Issue 12 December 2021

(C) All rights are reserved by Arun Muppliyath Raghavan. 